\title{
Early Warning for Tungsten Resources Industrial Security in China
}

\author{
Bing Gao ${ }^{1,}$, Zheng $\mathrm{Li}^{2, \mathrm{~b}}$ \\ ${ }^{1}$ Chinese Academy of Land and Resource Economics, Beijing 101149, China; \\ ${ }^{2}$ Beijing Geotechnical and Investigation Engineering Institute, Beijing 100083, China. \\ aMagaobintg@163.com, ${ }^{\mathrm{b}}$ lizheng1985911@sina.com
}

Keywords: China; Tungsten Resources; Healthy; Early warning.

\begin{abstract}
In order to accurately determine the healthy development of China's tungsten resources industry this paper proposed the main ideas of tungsten resources industry health warning system, then conducted a comprehensive analysis of the many factors that build health warning indicator system of tungsten resources industry, determine the critical point of tungsten resources industry health warning, and analyzed the early warning and forecast the warning degree. We can monitor and evaluate the China's tungsten resources production, consumption by the warning systems, and provide accurate data and analysis conclusions for the tungsten resources industry decision-making departments.
\end{abstract}

\section{Introduction}

China is the world's tungsten resources in most rich countries, is the world's largest producer of tungsten. But because of various reasons, a lot of problems in the process of development of tungsten industry in China, such as voice and pricing power loss, serious ecological environment destruction, the core technology of others, it is difficult to realize the sustainable and healthy development and needs to build health warning system of tungsten industry, provide decision basis for promoting the healthy development of tungsten industry in China.

\section{Summary of the healthy development of tungsten industry in China}

The tungsten industry itself is an open, dynamic artificial ecosystem, the interior of the tungsten industry from the perspective of the ecological system. It can be divided into industrial organization, industrial structure, industrial layout and industrial policy subsystem; The outside of the tungsten industry, can simply summed up in two parts of the natural ecological environment and social economic environment, the tungsten industry system and they exist between complex material, capital, information exchange. Using the concept of ecosystem health for Reference to the healthy development of tungsten industry refers to a country or a region of tungsten industry, external factors is in a state of normal development, under the function is integrated embodiment of its growth, order, stability and harmony.

\section{Main ideas of tungsten industry health warning}

Tungsten industry health warning is on the basis of industrial health evaluation, through the analysis of the related industrial economic data, the possible danger of tungsten industry forecast, and assist the related decision-making departments to formulate measures. Tungsten industry health warning mainly includes two aspects, one is the health of the tungsten industry forecasts, also is a kind of evaluation of industry health, secondly, it is to tungsten industry safety or health threats, timely warning signal for the industry. It is to establish a scientific and reasonable dynamic index system, according to a range of health warning, when critical range for tungsten industry relevant indicators, shall give an alarm to remind whether health prediction or industry security threat warning. 
The main methods of health warning in tungsten industry: (1) make sure early warning main indicators; (2) define early warning threshold, (3) the output results.

First of all, Based on the analysis of the threat to the healthy development of tungsten industry and the basis of internal and external factors, determine the main influencing index, and according to its influence degree, using relevant theory method, determine the index weight of tungsten industry health warning mark, forming a set of the early warning index system specification.

Secondly, considering the international practice, historical experience, expertise, etc factors, combined with the development of tungsten industry development of our country practice comprehensive consideration, determine the tungsten industry health warning or the single index results in dangerous and unhealthy, basic health, health and so on several levels. Warning threshold is dynamic change.

Finally is the output of the early warning result, it is the quantitative analysis of the result of early warning and response of early-warning index numerical change "p" in degree or intensity.

\section{The construction of a health warning index system of tungsten industry}

Healthy development of tungsten industry is a complex macroeconomic problems, cannot use a single index to measure, by means of the many factors influencing the tungsten production, supply and distribution to conduct a comprehensive analysis, find out the main indicators to determine.

\subsection{The content of the index system}

First, the tungsten total supply and demand rate is $\left(\mathrm{G}_{\mathrm{T}}-\mathrm{X}_{\mathrm{T}}\right) / \mathrm{X}_{\mathrm{T}}=\mathrm{L}_{\mathrm{gz}}$ where $\mathrm{G}_{\mathrm{T}}$ is the total supply of tungsten in the nation and XT is the national tungsten aggregate demand. The index for total supply tungsten and tungsten aggregate demand accounts for the difference between the total demands of the tungsten, the excess is negative for the shortage. Index can reflect the total supply tungsten deviate from the level of aggregate demand and tungsten overall safety level, at the same time, it combines the two aspects of supply and demand of tungsten, can be used as one of the main indicators of tungsten industry in China. Tungsten estimates of total supply: the initial inventory, prediction of current production, estimates that might be the sum of net imports of minus the necessary final inventory, get the current total supply of tungsten.

Second, the main variety of tungsten rate of the biggest difference of supply and demand is max $\left(\left|\left(G_{x}-X_{x}\right) / X_{x},\left(G_{y}-X_{y}\right) / X_{y},\left(G_{d}-X_{d}\right) / X_{d}\right|\right)=L_{x y d}$ where $\left(G_{x}, G_{y}, G_{d}\right)$ is the nationally representative respectively the total supply of tungsten products, respectively and the $\left(G_{x}, G_{y}, G_{d}\right)$ is the national representative tungsten products aggregate demand. Indicator reflects the rate of tungsten ore supply and demand balance of absolute value maximum.

Three, tungsten production volatility index is $\left(S_{t}-S_{t}^{\prime}\right) / S_{t}^{\prime}=\Delta S$ where $\left(S_{t}, S_{t}^{\prime}\right)$ is the actual production of tungsten respectively and trend of production. The index for tungsten accounts for the difference between the actual output and its trend yield trend yield percentage, is for the remaining, negative for the shortage, reflect a certain period of tungsten deviates from the value of the trend of practical production condition 7. The domestic tungsten production is a key part of the total supply tungsten and tungsten comprehensive production capacity of improved steadily is to enhance national tungsten available capacity, the basic way to realize sustainable tungsten industry. Tungsten production in China is to ensure that the main factors of tungsten ore supply and demand balance, and changes in years of tungsten production is bigger, is an important index of tungsten industry.

Fourth, tungsten demand volatility index is $\left(\mathrm{X}_{\mathrm{t}}-\mathrm{X}_{\mathrm{t}}^{\prime}\right) / \mathrm{X}_{\mathrm{t}}^{\prime}=\triangle \mathrm{X}$ where $\left(\mathrm{X}_{\mathrm{t}}, \mathrm{X}_{\mathrm{t}}^{\prime}\right)$ is the tungsten actual demand and trend of demand respectively. The index for tungsten accounts for the difference between the actual demand and its trend of demand trends demand percentage, reflects the current demand for tungsten may deviate from the demand of tungsten long-term trend. Tungsten requirements can be satisfied is an important aspect of measure whether the tungsten security. Total demand on the one hand may lead to the emergence of tungsten ore supply and demand gap, on the other hand may result in tungsten ore prices, will affect the sustainable and healthy development of tungsten industry. 
Five, the tungsten reserve requirements is $C_{t} / X_{t}=C X$ where $C_{t}$ is the tungsten reserves. Tungsten reserves including national reserves, corporate and local reserves. Tungsten tungsten reserves for mediation we adopted, alleviate the impact of the equilibrium of supply and demand of tungsten production fluctuation of tungsten is of great importance ${ }^{[8]}$. Tungsten reserve ratios is important parameters of the factors affecting the healthy development of tungsten industry.

Six, tungsten price volatility index is $\left(\mathrm{P}_{\mathrm{t}}-\mathrm{P}_{\mathrm{t}-1}\right) / \mathrm{P}_{\mathrm{t}-1}=\triangle \mathrm{P}$ where $\mathrm{P}_{\mathrm{t}}$ is the current price of tungsten. Tungsten price change index is a certain period, this period of tungsten ore price minus on the issue of tungsten ore price and on the ratio of the issue price of tungsten. Under the condition of the market more perfect, the tungsten market price fluctuations can reflect the change of tungsten ore supply and demand balance, therefore, tungsten price rate of change in the very great degree can directly reflect the tungsten industry problems, and can be used as a measure of tungsten industry in the early warning system of tungsten industry must be special attention of tungsten ore prices. Constitute a relatively stable price is one of the basic conditions for sustainable tungsten industry.

Seven, the tungsten resources bearing capacity coefficient is $\mathrm{Q} /(\mathrm{M} \times \mathrm{T})=\mathrm{Pi}$ where $\mathrm{Q}$ is the tungsten resources available reserves and $\mathrm{M}$ is the said tungsten resources consumption and $\mathrm{T}$ is the fixed number of year for reasonable development. Change index said existing tungsten resources available to support the development of national economy, this index is not stagnant index, with the development of national economy, tungsten resources bear ability, preliminary analysis of the future demand supply cycle index.

\subsection{The weight of the index system}

Health warning research of tungsten industry is to provide the theory and practice basis the focus of the formulation and the development of specific industry and tungsten industry development policy in china. In the process of concrete research, need according to the tungsten industry development present situation of tungsten industry health warning related index weight assignment, in order to improve the scientific nature and reliability of the calculation results. In this paper, we adopt the Delphi method and entropy weight method. First invited a number of tungsten industry experts weights of index weights are given respectively, and calculate the average, then the results back to the experts. Because of using the Delphi method, it is concluded that the weight of subjectivity is stronger, in order to make the health warning index weight tungsten industry has a certain objectivity, entropy weight method can be used to modify the above weight, finally combine both get the combination of the subjective and objective weights. Entropy weight method is based on health index to determine the index weight of the objects of warning a kind of method, reflects the comparative relationship between the indexes, the calculation process is no longer here.

\section{The implementation of Chinese tungsten industry health warning system}

\subsection{Health warning index critical value determination of tungsten industry}

Tungsten industry health warning of individual indicators point set is the key of the early warning model, the indicators for accurate monitoring changes in the situation, the operation condition of the whole tungsten industry make the right judgment, the current application more widely in the following ways: the standard method, literature review method, extremes method (average method) and expert evaluation method and comprehensive analysis method. Because tungsten industry is our country the advantage of industry, determine the health threshold for a single metric in conjunction with the development of comprehensive consideration of international and domestic tungsten mining industry may be, taking into account current development tungsten mining industry, dynamically adjusting the threshold of each index.

\subsection{Mapping tungsten mining industry health warning indicators point value}

Respectively, will be satisfied with a single upper limit value of early warning indicators, it does not allow the lower limit value, the value of each index calculation map, depending on the type of early warning indicators can be divided into the following. The index value the greater the health indicators is $\mathrm{X}_{\text {upper }}+\left(\mathrm{Q}-\mathrm{Y}_{\text {lower }}\right) \times\left(\mathrm{X}_{\text {upper }}-\mathrm{X}_{\text {lower }}\right) /\left(\mathrm{Y}_{\text {upper }}-\mathrm{Y}_{\text {lower }}\right)=\mathrm{P}_{\mathrm{i}}$. The smaller the index value of the health indicator is $\mathrm{X}_{\text {lower limit }}-\left(\mathrm{Q}-\mathrm{Y}_{\text {lower }}\right) \times\left(\mathrm{X}_{\text {upper }}-\mathrm{X}_{\text {lower }}\right) /\left(\mathrm{Y}_{\text {upper }}-\mathrm{Y}_{\text {lower }}\right)=\mathrm{P}_{\mathrm{i}}$. Health index value at a 
certain point, a point away from unhealthy indicators is $2 \times\left|\mathrm{Q}-\left(\mathrm{Y}_{\text {upper }}-\mathrm{Y}_{\text {lower }}\right) / 2\right| \times\left(\mathrm{X}_{\text {upper }}-\mathrm{X}_{\text {lower }}\right) /\left(\mathrm{Y}_{\text {upper }}-\mathrm{Y}_{\text {lower }}\right)=\mathrm{P}_{\mathrm{i}}$.In the above formula: $\mathrm{P}_{\mathrm{i}}$ is mapping the value pointed to target, $\mathrm{Q}$ is actual value point target, $\mathrm{X}_{\text {upper }}$ refers to the health status online, $\mathrm{X}_{\text {lower }}$ refers to the state of health, the $\mathrm{Y}_{\text {upper }}$ refers to the upper warning limit, $\mathrm{Y}_{\text {lower }}$ refers to the warning limit the lower limit.

According to the above formula to calculate the individual indicators health score value mapping, according to the corresponding weight to calculate the tungsten industry health warning index $\mathrm{K}$, its computation formula is $\sum \mathrm{P}_{\mathrm{i}} \mathrm{W}_{\mathrm{i}}=\mathrm{K}$ Where $\mathrm{P}_{\mathrm{i}}$ is the index map values and $\mathrm{W}_{\mathrm{i}}$ is the index weight.

\subsection{Tungsten industry health warning results output}

In order to have a number of tungsten industry health knowledge, we can to classification of tungsten industry health status or degree, which divides into the tungsten industry health risk, health, basic health, four levels, respectively the index interval [0-0.2], (0.2-0.5], (0.5-0.8], (0.8-1].

\section{Conclusion}

China is a country that good at tungsten resources, has the comprehensive advantages of development of tungsten industry, but it is not strong enough in tungsten industry and faces many threats. Through the establishment and operation of health warning system for tungsten industry, it can tungsten resource production, storage, distribution, consumption, etc. for monitoring and evaluation, to provide accurate data for tungsten industry data and analysis, from the macroscopic and microcosmic aspects to promote the healthy development of tungsten industry in China.

\section{References}

[1]. Li Menggang. Industrial security theory research [M], Beijing: economic science press, 2010

[2]. Hong Ming Yang. The present situation and sustainable development strategic decisions of mining and utilizing tungsten resources of china[J], China Tungsten Industrial, 2004,19(5):25-28

[3]. China mining network. The sketch map of mineral resource distribution of P.r.china (tungsten resources),[DB/OL].2012-8-11,2012-06-20.

ttp://www.mine168.com/XXziliao.asp?zziliaoID=20111

[4]. Xu Fangdeng. Industrial security of ecological early warning mechanism research [M]. Beijing: science press, 2010

[5]. Zhu LiMeng. Import and Export of Chinese Agriculture Products and Early-warning Analysis on Agriculture Security[J]. Finance \& Economics, 2007,6(23):111-116 